- Additional material is published online only. To view please visit the journal online (http://dx.doi.org/10.1136/ bjsports-2019-100885).

For numbered affiliations see end of article.

\section{Correspondence to} Dr Alex Scott, Department of Physical Therapy, University of British Columbia, Vancouver V6T 1Z4, Canada: ascott@mail.ubc.ca

Accepted 30 June 2019 Published Online First 9 August 2019
Check for updates

(C) Author(s) (or their employer(s)) 2020. No commercial re-use. See rights and permissions. Published by BMJ.

To cite: Scott $A$, Squier $\mathrm{K}$, Alfredson $\mathrm{H}$, et al. Br J Sports Med 2020:54:260-262.

\title{
ICON 2019: International Scientific Tendinopathy Symposium Consensus: Clinical Terminology
}

\author{
Alex Scott (D) ,' Kipling Squier, ${ }^{1}$ Hakan Alfredson (D) ,' ${ }^{2}$ Roald Bahr, ${ }^{3}$ Jill L Cook, \\ Brooke Coombes, ${ }^{5}$ Robert-Jan de Vos, ${ }^{6}$ Siu Ngor Fu, ${ }^{7}$ Alison Grimaldi, ${ }^{8}$ \\ Jeremy S Lewis (D) , ${ }^{9}$ Nicola Maffulli, ${ }^{10}$ SP Magnusson, ${ }^{11}$ Peter Malliaras, ${ }^{12}$ \\ Sean Mc Auliffe, ${ }^{13}$ Edwin H G Oei, ${ }^{14}$ Craig Robert Purdam, ${ }^{15}$ Jonathan D Rees (D) , 16,17 \\ Ebonie Kendra Rio, ${ }^{4}$ Karin Gravare Silbernagel (D) , ${ }^{18}$ Cathy Speed, ${ }^{19,20}{ }^{2}$ Adam Weir, ${ }^{21}$ \\ Jennifer Moriatis Wolf, ${ }^{22}$ Inge van den Akker-Scheek, ${ }^{23}$ Bill T Vicenzino (D) , ${ }^{24}$ \\ Johannes Zwerver (D) 25
}

\section{BACKGROUND}

Persistent tendon pain that impairs function has inconsistent medical terms that can influence choice of treatment. ${ }^{1}$ When a person is told they have tendinopathy by clinician A or tendinitis by clinician B, they might feel confused or be alarmed at receiving what they might perceive as two different diagnoses. This may lead to loss of confidence in their health professional and likely adds to uncertainty if they were to search for information about their condition. Clear and uniform terminology also assists inter-professional communication. Inconsistency in terminology for painful tendon disorders is a problem at numerous anatomical sites.

Historically, the term 'tendinitis' was first used to describe tendon pain, thickening and impaired function (online supplementary figure S1). The term 'tendinosis' has also been used in a small number of publications, some of which were very influential. ${ }^{23}$ Subsequently, 'tendinopathy' emerged as the most common term for persistent tendon pain. $^{45}$ To our knowledge, experts (clinicians and researchers) or patients have never engaged in a formal process to discuss the terminology we use. We believe that health professionals have not yet agreed on the appropriate terminology for painful tendon conditions.

The authors of this paper, a group of international clinical and research experts from different disciplines, aimed to achieve a consensus in terminology for persistent tendon disorders. We ran a Delphi and consensus process that culminated in a face-to-face meeting at the fifth International Scientific Tendinopathy Symposium (ISTS) in Groningen, the Netherlands, on 26 September 2018 (placeholder for sentence about the other two papers and accompanying editorial if they get accepted). Here, we present the resulting consensus statements on terminology for persistent tendon pain.

\section{METHODS}

Our two-stage Delphi design, consensus process consisted of an online survey followed by a faceto-face meeting. One of us (AS) drafted 11 statements about terminology relevant to typical clinical presentations and clinical findings. Following several teleconferences by the project committee (AS, BV, HZ, ER, AW and SM), these statements were further refined. The role of the committee members was to develop and execute the Delphi process. The final document we sent out for Stage 1 of the consensus process (see below) featured 10 statements (online supplementary appendix 2).

\section{Stage 1}

We e-mailed invitations to 30 tendinopathy experts. These experts were unanimously selected by the core author group, according to four goals:

1. Encourage participation by both men and women or male and female experts;

2. Choose from among top ranked experts (Web of Science and Expertscape searches) who have published original research on tendinopathy;

3. Achieve representation from multiple disciplines (sports physicians, rheumatologists, physiotherapists, epidemiologists, clinicians, researchers, surgeons, radiologists);

4. Have broad geographical representation.

The invitation letter explained the process and the rules for participation, including an option to participate in both the online survey and the in-person meeting, or the online survey alone (online supplementary appendix 3). Of 30 invitees, 28 people with a clinical and/or research interest responded and agreed to participate. Of the two who did not agree to participate, one did not respond within the time frame and one withdrew after agreeing to participate due to personal reasons, unrelated to the survey. The experts were sent a link to an anonymised survey (Survey Monkey, San Mateo, CA, USA). For each statement, the experts could choose from agree, disagree or don't know/ not sure, as well as an optional space for comments. One author (BV) compiled the survey results and shared them with the core author group for analysis. Statements that achieved consensus $(70 \%$ or higher agreed or disagreed) were not discussed further (online supplementary appendices 4 and 5).

\section{Stage 2}

Five of the project committee members (AS, BV, $\mathrm{HZ}, \mathrm{ER}$ and SM) and 10 of the experts who also 
Box 1 ICON 2019: international scientific tendinopathy symposium consensus: clinical terminology

- Tendinopathy is the preferred term for persistent tendon pain and loss of function related to mechanical loading.

- Tendon tear (partial or complete) refers to a macroscopic* discontinuity of a load-bearing tendon.

- Imaging is not always necessary for a diagnosis of tendinopathy.

- Patellar tendinopathy is the preferred term for persistent patellar tendon pain and loss of function related to mechanical loading.

- Achilles tendinopathy is the preferred term for persistent Achilles tendon pain and loss of function related to mechanical loading.

- Peroneal (fibularis) tendinopathy is the preferred term for persistent peroneal (fibularis) tendon pain and loss of function related to mechanical loading.

- Persistent tendon pain and loss of function related to mechanical loading of the medial or lateral elbow tendons should be referred to as medial or lateral elbow tendinopathy.

*Large enough to be visible without magnification.

completed the survey attended the in-person meeting in Groningen (15 participants). All participants funded their own travel and accommodation and received no payments for attending. We distributed a summary report of the survey results to attendees before the in-person meeting. This report included response rates for each question and free-text entries (online supplementary appendix 5)

At the meeting, AS facilitated an open discussion using Power Point to display the five statements about which online participants $(n=28)$ had not achieved consensus after the online survey. After discussion, the group excluded two statements from further analysis due to the possibility of perceived lack of diverse disciplinary representation at the in-person group (\#5, imaging, and $\# 10$, rotator cuff). For the other statements, a vote was cast by a secret ballot. At this step, the voting options were yes/agreed or no/disagreed. The votes were counted by two individuals in the room and the results were announced and recorded (online supplementary appendix 6).

A draft of the manuscript was circulated to all 28 authors and all comments/edits were accepted. Three experts declined to be listed as a co-author on the manuscript, and communicated this to one of us by email (BV). Each of them stated they did not disagree with the statements, but did not feel they had contributed enough to warrant authorship.

\section{RESULTS}

The ICON 2019: ISTS Consensus on Clinical Terminology is shown in box 1 .

\section{DISCUSSION}

Tendinopathy is the preferred term for persistent tendon pain and loss of function related to mechanical loading

This definition of tendinopathy emphasises pain and function. Using the term tendinopathy can help frame treatment and improve communication between professionals and patients. With this statement we hope that the term tendinopathy will continue to supersede other terms. The term does not imply the presence of a particular pathological or biochemical process which cannot routinely be assessed clinically.

\section{Tendinosis: a label without a disease?}

We did not agree on the statement that tendinosis refers to a loss of microstructure (as opposed to macroscopic tearing). Tissue changes seen on imaging or histology and labelled as tendinosis may represent a snap-shot of a number of morphological processes with uncertain clinical relevance. In some situations, a loss of collagen alignment could be physiological (eg, as a result of ageing, adaptation or repair) rather than pathological. We cannot recommend using the term tendinosis unless future work can establish a consensus on its definition.

Tendon tear (partial or complete) refers to a macroscopic discontinuity of load-bearing tendon.

Tears represent a clinical entity for some tendons which is distinct from tendinopathy, and which may require different management to that of tendinopathy. The ICON group defined tears as a macroscopic (as opposed to microscopic) discontinuity of load-bearing tendon. Future research should examine whether tears, partial tears and tendinopathy can be reproducibly distinguished. If they could be, it may have clinical utility (ie, guide treatment).

We need to discover whether diagnosing partial tears leads to improved patient outcomes, and in which situations it adds no value. We believe there is not a validated means of identifying partial tears on imaging for all tendons.

\section{Does a tendinopathy at each region in the body warrant its own name?}

Tendon pain occurs in several anatomical sites such as (but not limited only to) the Achilles, patellar, peroneal (fibularis) and elbow. We did not address nomenclature at other tendons, such as tibialis posterior or gluteal tendons.

The term 'rotator cuff tendinopathy' was on our list of options but the group did not achieve consensus on that term as being ideal for shoulder pain and loss of function because a proportion of participants felt that the diagnosis could not be made clinically due to the variety of nociceptive structures near to the rotator cuff tendons. With respect to this, the Groningen statement is consistent with previous Delphi studies and clinical guidelines which include tendinopathy as part of a spectrum of subacromial or rotator-cuffrelated shoulder pain and injury. ${ }^{6-8}$ Those guidelines recommend the terms subacromial pain (or impingement) syndrome for patients with painful shoulder tendons and loss of function; the term rotatorcuff-related shoulder pain has also been proposed. ${ }^{8}$

\section{Future work}

Imaging can provide additional information which can assist with diagnosis. If terminology is needed to describe morphological tendon changes as seen with imaging or histology, we recommend that clinicians use objective measures (if their validity and reliability can be demonstrated) such as increased or decreased signal intensity (for MR images), collagen disorientation or hypoechogencity (in the case of ultrasound images), etc. An ISTS imaging subgroup was formed to explore/consider issues related to the role of imaging for diagnosis in for tendinopathy and relevant terminology.

\section{CONCLUSION}

We recommend that clinicians and patients use the term tendinopathy to refer to persistent tendon pain related to mechanical loading. 


\section{Author affiliations}

'Department of Physical Therapy, University of British Columbia, Vancouver, British Columbia, Canada

2Department of Integrative Medical Biology, Umeå University, Umeå, Sweden

${ }^{3}$ Department of Sports Medicine, Oslo Sports Trauma Research Center, Norwegian School of Sport Sciences, Oslo, Norway

${ }^{4}$ La Trobe Sport and Exercise Medicine Research Centre, La Trobe University, Melbourne, Victoria, Australia

${ }^{5}$ Department of Allied Health Sciences, Physiotherapy, Griffith University, Nathan, Queensland, Australia

${ }^{6}$ Department of Orthopaedics and Sports Medicine, Erasmus University Medical Centre, Rotterdam, The Netherlands

${ }^{7}$ Department of Rehabilitation Sciences, The Hong Kong Polytechnic University, Kowloon, Hong Kong

${ }^{8}$ School of Health and Rehabilitation Sciences, University of Queensland, Brisbane, Queensland, Australia

${ }^{9}$ Department of Allied Health Professions, University of Hertfordshire, Hatfield, UK

${ }^{10} \mathrm{Centre}$ for Sports and Exercise Medicine, Barts and The London School of Medicine and Dentistry, London, UK

${ }^{11}$ Department of Clinical Medicine, Bispebjerg-Frederiksberg Hospital, Copenhagen, Denmark

${ }^{12}$ Department of Physiotherapy, Monash University Faculty of Medicine Nursing and Health Sciences, Frankston, Victoria, Australia

${ }^{13}$ Qatar Orthopaedic and Sports Medicine Hospital, Doha, Qatar

${ }^{14}$ Department of Radiology \& Nuclear Medicine of Erasmus MC, University Medical Center, Rotterdam, The Netherlands

${ }^{15}$ Department of Physical Therapies, Australian Institute of Sport, Bruce, Australian Capital Territory, Australia

${ }^{16}$ Department of Rheumatology, Cambridge University Hospitals NHS Foundation Trust, Cambridge, UK

${ }^{17}$ Headley Court, Defence Medical Rehabilitation Centre, Surrey, UK

${ }^{18}$ Department of Physical Therapy, University of Delaware, Newark, Delaware, USA

${ }^{19}$ Department of Rheumatology/Sports Medicine, Cambridge University Hospital, Cambridge, UK

${ }^{20}$ Cambridge Lea Hospital, Cambridge, UK

${ }^{21}$ Department of Sports Medicine, Aspetar hospital, Doha, Qatar

${ }^{22}$ Department of Orthopaedic Surgery, University of Connecticut, Farmington,

Connecticut, USA

${ }^{23}$ Department of Sports Medicine, University Medical Center Groningen, University of Groningen, Groningen, The Netherlands

${ }^{24}$ Department of Physiotherapy, University of Queensland, Brisbane, Queensland, Australia

${ }^{25}$ Department of Sports Medicine, University Medical Center, Groningen, The Netherlands
Contributors AS: study idea, methods, analysis, writing first draft, compiling contributing authors' comments. BV: methods, data collection and analysis, writing. JZ, ER, AW and SM: methods, writing. All others: Delphi participation, writing.

Funding The authors have not declared a specific grant for this research from any funding agency in the public, commercial or not-for-profit sectors.

Competing interests None declared.

Patient consent for publication Not required.

Provenance and peer review Not commissioned; externally peer reviewed.

\section{ORCID iDs}

Alex Scott http://orcid.org/0000-0003-0366-8404

Hakan Alfredson http://orcid.org/0000-0003-4109-7606

Jeremy S Lewis http://orcid.org/0000-0001-7870-9165

Jonathan D Rees http://orcid.org/0000-0002-5477-8358

Karin Gravare Silbernagel http://orcid.org/0000-0001-7566-407X

Bill T Vicenzino http://orcid.org/0000-0003-0253-5933

Johannes Zwerver http://orcid.org/0000-0002-8499-2806

\section{REFERENCES}

1 Nickel B, Barratt A, Copp T, et al. Words do matter: a systematic review on how different terminology for the same condition influences management preferences. $B M J$ Open 2017;7:014129.

2 Kraushaar BS, Nirschl RP. Tendinosis of the elbow (tennis elbow). clinical features and findings of histological, immunohistochemical, and electron microscopy studies. J Bone Joint Surg Am 1999;81:259-78.

3 Kannus P, Józsa L. Histopathological changes preceding spontaneous rupture of a tendon. A controlled study of 891 patients. J Bone Joint Surg Am 1991;73:1507-25.

4 Khan KM, Cook JL, Kannus P, et al. Time to abandon the "tendinitis" myth. BMJ 2002;324:626-7.

5 Maffulli N, Khan KM, Puddu G. Overuse tendon conditions: time to change a confusing terminology. Arthroscopy 1998;14:840-3.

6 Diercks R, Bron C, Dorrestijn 0, et al. Guideline for diagnosis and treatment of subacromial pain syndrome: a multidisciplinary review by the Dutch orthopaedic association. Acta Orthop 2014;85:314-22.

7 Huisstede BMA, Miedema HS, Verhagen AP, et al. Multidisciplinary consensus on the terminology and classification of complaints of the arm, neck and/or shoulder. Occup Environ Med 2007:64:313-9.

8 Lewis J. Rotator cuff related shoulder pain: assessment, management and uncertainties. Man Ther 2016:23:57-68. 